\title{
ON MEASURE IN ABSTRACT SETS
}

\author{
BY \\ DOROTHY MAHARAM \\ INTRODUCTION
}

In their paper $\left.{ }^{1}\right)$ Sur les décompositions dénombrables Banach and Tarski obtained a result which can be restated as follows:

A necessary and sufficient condition that two Lebesgue measurable, euclidean sets $\boldsymbol{a}$ and $\boldsymbol{a}^{\prime}$, shall have equal measure is that there exist null sets $\boldsymbol{n}<\boldsymbol{a}$ and $\boldsymbol{n}^{\prime}<\boldsymbol{a}^{\prime}$, such that $\boldsymbol{a}-\boldsymbol{n}$ and $\boldsymbol{a}^{\prime}-\boldsymbol{n}^{\prime}$ are the respective unions of sequences of disjoint measurable sets, of which corresponding sets are congruent.

If we then identify sets differing on only a null set, there exists a class of transformations on the measurable sets such that two sets are of equal measure if and only if they correspond under some transformation of that class. Then just as the elementary notion of the volume of an $n$-dimensional interval is generalized to that of any measure function on a Borel field, so the equally elementary notion of equality of volume, defined for these figures by the relation congruence, can be generalized to the notion of equality of measure defined for some field of sets by a suitable class of transformations. This is done as follows:

In Part I, we consider a complemented, distributive $\sigma$-lattice $M$ with a zero element, and a class $\Phi$ of $\sigma$-isomorphisms on the principal ideals of $M$. The lattice $M$ is to be taken to correspond to a family of measurable sets modulo the null sets, and $\Phi$ as a semi-group of measure-preserving transformations. Then $\Phi$ generates an equivalence relation $a \sim b$ between elements of $M$ which is countably additive and hereditary in the sense that $a \sim b$, $a^{\prime}<a$ imply that there exists a $b^{\prime}<b$ such that $a^{\prime} \sim b^{\prime}$. It is then shown for the bounded elements of $M$, that is, those which are not equivalent to any proper subelement, that the relation $a \sim b$ is also preserved by subtraction and by taking limits of monotonic sequences. It may be remarked that these latter results yield an independent proof $\left({ }^{2}\right)$ of the theorem of Banach and Tarski. A construction is also given leading to a definition of a complete measure. In Part II, the measure of an element of $M$ is defined as the totality of its equiva-

Presented to the Society February 22, 1941; received by the editors November 19, 1940, and in revised form, March 15, 1941.

(1) Fundamenta Mathematicae, vol. 6 (1924), pp. 244-277. The theorem quoted is on page 277. This result has been extended to the case of the Haar measure on a locally compact group by Professor John von Neumann in a series of lectures at the Institute for Advanced Study.

(2) See Example 15.3 for a proof in a generalized form. 
lent elements, that is, of images under $\Phi$; and a partial ordering and an operation of addition are defined in the set of measures. A necessary and sufficient condition is then given that the set of measure values be order and addition isomorphic to a set of positive numbers. Such an isomorphism is necessarily unique, up to a multiplicative constant. These results will be analyzed in detail in another paper. In Part III, a sufficient condition is given that, for a family of sets upon which a numerical measure is defined, two sets of equal measure shall correspond under some measure-preserving transformation, and so that the given measure shall be derivable by the procedure of Part II. It turns out that this is the case for a measure on a separable Borel field, provided there are no minimum sets of positive measure. However, a trivial generalization of the theory of Part II can be seen to be applicable to any measure function.

\section{PART I}

1. Ideals and isomorphisms in the lattice $M$. Let $M \equiv\{a, b, c, \cdots\}$ be a complemented distributive $\sigma$-lattice with a zero element; i.e., satisfying the system $(\alpha)$ :

i. $M$ has a partial ordering $a<b$ ( $b$ contains $a$ ) such that $a<b$ excludes $a=b$.

ii. Every sequence $\left(a_{n}\right) \subseteq M$ has a supremum and an infimum $\sum a_{n}$ and $\prod a_{n}$, respectively. If $\left(a_{n}\right)$ is monotone increasing (decreasing), we denote $\sum a_{n}\left(\prod a_{n}\right)$ by $\lim a_{n}$. We also write $a+b$ and $a b$ for the supremum and infimum of $(a, b)$.

iii. $M$ has a zero element $\theta$. Any elements $a, b$ such that $a b=\theta$ are called disjoint.

iv. If $a<b$, then there exists a unique element, which will be written $b-a$, such that $(b-a) a=\theta$, and $a+(b-a)=b$.

v. For any sequence $\left(a_{n}\right)$ and any $a, a\left(\sum a_{n}\right)=\sum a a_{n}$.

1.1. Definition. A class $M^{\prime} \subseteq M$ is called a $\sigma$-ideal if $a^{\prime}<a \in M^{\prime}$ implies that $a^{\prime} \in M^{\prime}$, and if $\left(a_{n}\right) \subset M^{\prime}$ implies that $\sum a_{n} \in M^{\prime}$. The ideal $M^{\prime}$ is called principal if there exists an element a such that $a^{\prime} \in M^{\prime}$ if and only if $a^{\prime} \leqq a$. The principal ideal corresponding to an element a will be written $I(a)$.

1.2. Definition. A univocal correspondence $\phi$ defined on a $\sigma$-ideal $M^{\prime} \subseteq M$ onto a $\sigma$-ideal $M^{\prime \prime} \subseteq M$ is called a $\sigma$-isomorphism if for every sequence $\left(a_{n}\right) \subset M$, $\phi\left(\sum a_{n}\right)=\sum \phi\left(a_{n}\right)$, and $\phi\left(\prod a_{n}\right)=\prod \phi\left(a_{n}\right)$. The correspondence inverse to $\phi$ is also a $\sigma$-isomorphism which will be written $\phi^{-1}$.

1.21. For any $\sigma$-isomorphism $\phi, \phi(a)=\theta \equiv a=\theta ; a^{\prime}<a \equiv \phi\left(a^{\prime}\right)<\phi(a)$, and $\phi\left(a-a^{\prime}\right)=\phi(a)-\phi\left(a^{\prime}\right)$.

1.22. Let $m$ be a $\sigma$-ring of subsets of a set $s ; n$, a $\sigma$-ideal in $m$; and $M$, the residue class ring $m / n$. Then any univocal point correspondence between the subsets $\boldsymbol{a}$ and $\boldsymbol{b}$ in $m$ which leaves the rings $m$ and $n$ invariant leads to a $\sigma$-isomorphism of the principal ideals in $M$ corresponding to $\boldsymbol{a}$ and $\boldsymbol{b}$. 
1.23. Definition. Let $\left(\phi_{n}\right)$ be a sequence of $\sigma$-isomorphisms between the principal ideals $I\left(a_{n}\right)$ and $I\left(b_{n}\right)$. If the sequences $\left(a_{n}\right)$ and $\left(b_{n}\right)$ are each disjoint, then $\left(\phi_{n}\right)$ is said to have a sum $\sum \phi_{n}$; namely, the isomorphism $\phi\left(a^{\prime}\right)$ defined for $a^{\prime} \leqq \sum a_{n}$ by $\phi\left(a^{\prime}\right)=\sum \phi_{n}\left(a^{\prime} a_{n}\right)$.

2. The class $\Phi$ of $\sigma$-isomorphisms. Equivalence. Boundedness. Let $\Phi$ be a class of $\sigma$-somorphisms each defined between principal ideals of $M$, and satisfying $(\beta)$ :

i. The inverse of any isomorphism in $\Phi$ is in $\Phi$.

ii. If $\phi, \phi^{\prime}$ are in $\Phi$, and are defined on $I(a), I\left(a^{\prime}\right)$, respectively, then the resultants $\phi \phi^{\prime}$ and $\phi^{\prime} \phi$, defined on a $\phi^{\prime}\left(a^{\prime}\right)$ and $a^{\prime} \phi(a)$, are in $\Phi$.

iii. If $\left(\phi_{n}\right) \subset \Phi$, and if $\sum \Phi_{n}$ exists, then $\sum \phi_{n} \in \Phi$.

iv. At least one isomorphism of $\Phi$ is defined on any principal ideal of $M$.

2.1. Definition. Two elements of $M$ are called $\Phi$-equivalent if they correspond under some isomorphism of $\Phi$. This relation will be written $a \sim b$.

2.2. The relation $\Phi$-equivalence has the following properties:

i. It is an equivalence relation, and so partitions $M$ into a set of exhaustive and mutually exclusive classes $M_{\alpha}$. The unique class containing an element $a$ may be denoted by $M(a)$. Obviously, $M(a)=M\left(a^{\prime}\right)$ if and only if there is an isomorphism $\phi$ of $\Phi$ such that $a^{\prime}=\phi(a)$.

ii. If $\left(a_{n}\right),\left(b_{n}\right)$ are sequences of disjoint elements of $M$ such that $a_{n} \sim b_{n}$, then $\sum a_{n} \sim \sum b_{n}$; i.e., $\Phi$-equivalence is countably additive.

iii. It is hereditary in the sense that if $a \sim b$, and $a^{\prime}<a$, then there exists $a$ $b^{\prime}<b$ such that $a^{\prime} \sim b^{\prime}$.

2.3. Definition. An element $a$ of $M$ is unbounded (with respect to $\Phi$ ) if there exists an element $a^{\prime}<a$ such that $a \sim a^{\prime}$, and $a-a^{\prime} \neq \theta$. Otherwise $a$ is bounded.

2.31. i. If $a$ is bounded and $a^{\prime}<a$, then $a^{\prime}$ is bounded. ii. If $a \sim a+b$, and $b-a b \neq \theta$, then $a$ is unbounded. iii. If $a \sim a^{\prime}$, then $a$ and $a^{\prime}$ are bounded or unbounded together.

2.32. Definition. A class $M_{\alpha}$ of equivalent elements of $M$ is bounded or unbounded according as its elements are either all bounded or all unbounded.

2.33. Theorem. A necessary and sufficient condition that an element a be unbounded is that a contain a sequence of disjoint equivalent elements $a_{n} \neq \theta$.

Proof. Suppose such a sequence exists. Then by 2.2 , ii, $\sum a_{n} \sim \sum a_{2 n}$; and since $\sum a_{2 n-1} \neq \theta, \sum a_{n}$ is unbounded. Conversely, if $a$ is unbounded, there exists an element $a^{\prime}<a$ and an isomorphism $\phi$ of $\Phi$ such that $a^{\prime}$ and $a-a^{\prime}$ are different from $\theta$ and $\phi(a)=a-a^{\prime}$. Then an infinite sequence of elements satisfying the required conditions can be defined inductively by the formula $a_{1}=a$, and for $n>1, a_{n}=\phi\left(a_{n-1}\right)$. 
3. Equivalence of complements of bounded equivalent elements. The results of this section can be summarized as follows:

If $a \sim b$, and if either (i) there exists a $\sigma$-isomorphism $\phi$ of $\Phi$ under which $a b$ is invariant, or (ii) the elements $a$ and $b$ are bounded, then for any $c>(a+b)$, $c-a \sim c-b$.

The assertions leading to this statement are the following:

3.1. A necessary and sufficient condition that for any element $c \geqq(a+b)$, $c-a \sim c-b$, is that $a-a b \sim b-a b$. The necessity is obvious, and the sufficiency follows from the fact that $c-(a+b) \sim c-(a+b)$ (identity), and $a-a b \sim b-a b$ (by hypothesis). Since the terms on either side are disjoint, their respective sums are equivalent and $c-a \sim c-b$.

3.2. Theorem. If $a \sim b$, and $a$ and $b$ are bounded, then $a-a b \sim b-a b$.

The general argument of the proof is as follows: First it is shown that there exist sequences $\left(a_{n}\right)$ and $\left(b_{n}\right)$ of elements of $M$ contained in $a-a b$ and $b-a b$, respectively, such that $\phi^{n}\left(a_{n}\right)<a b$, and $\phi^{n+1}\left(a_{n}\right)=b_{n}$. From this it is verified that both families are disjoint. Finally it is shown that $\sum a_{n}=a-a b$ and $\sum b_{n}=b-a b$, which proves the theorem.

Let us first restate this in the more convenient form:

If $a+x \sim b+x$, where $a+x, b+x$ are bounded, and $a x=b x=\theta$, then $a \sim b$.

Proof. We have given that there exists an isomorphism $\phi$ of $\Phi$ such that $\phi(a+x)=b+x$. We shall write this as

$$
a+x \sim b+x(\phi) .
$$

Then

$$
a \phi^{-1}(b) \sim b \phi(a) .
$$

By taking differences on both sides we get

$$
a \phi^{-1}(x)+x \sim b \phi(x)+x .
$$

If we can show that

$$
a \phi^{-1}(x) \sim b \phi(x),
$$

then by summing corresponding sides of (2) and (4), we get the required result. Now let

$$
\begin{aligned}
& a_{0} \equiv a \phi^{-1}(x) \equiv a-a \phi^{-1}(b), \\
& b_{0} \equiv b \phi(x) \equiv b-b \phi(a), \\
& x_{0} \equiv \phi\left(a_{0}\right) \leqq x
\end{aligned}
$$

Since $\phi$ is defined on $x \leqq a+b$, we can define inductively for $n>0$

$$
x_{n} \equiv x \phi\left(x_{n-1}\right) \text {. }
$$

It can be shown by induction that the elements $a, x_{0}, x_{1}, \cdots$, are dis 
joint. For since $a_{0} \leqq a$, and $x_{0} \leqq x, a_{0}$ and $x_{0}$ are disjoint. Suppose that $a_{0}, x_{0}, \cdots, x_{n-1}$ are disjoint. But for $0<k \leqq n, \phi^{-1}\left(x_{k}\right) \leqq x_{k-1}$, and $\phi^{-1}\left(x_{0}\right)=a_{0}$. Hence for $0 \leqq k \leqq n$, the elements $\phi^{-1}\left(x_{k}\right)$, and so also the elements $x_{k}$, are disjoint by the hypothesis of the induction, which is now complete. We define

$$
b_{n}=b \phi\left(x_{n-1}\right), \quad n=1,2,3, \cdots \text {. }
$$

Since $\phi^{-1}\left(b_{n}\right) \leqq x_{n-1}$, it follows that the elements $b_{n}$ are disjoint. Further, since $\phi\left(x_{n}\right) \leqq \phi(x) \leqq b+x$,

$$
\phi\left(x_{n-1}\right)=x \phi\left(x_{n-1}\right)+b \phi\left(x_{n-1}\right)=x_{n}+b_{n},
$$

that is,

$$
x_{n-1} \sim x_{n}+b_{n}(\phi) \quad \text { for } n=1,2,3, \cdots
$$

and we also have

$$
a_{0} \sim x_{0}(\phi) .
$$

Since the terms on the right (the left) side of (9) and (10) are disjoint, their respective sums are equivalent and

$$
\left(a_{0}+\sum_{0}^{\infty} x_{n}\right) \sim\left(\sum_{0}^{\infty} x_{n}+\sum_{1}^{\infty} b_{n}\right)(\phi) .
$$

But from (3) and (5)

$$
a_{0}+x \sim b_{0}+x(\phi),
$$

and for $n \geqq 1, b_{n} \leqq b \phi(x)=b_{0}$, whence the respective differences of corresponding sides of (11) and (12) are equivalent; that is,

$$
x-\sum_{0}^{\infty} x_{n} \sim\left(b_{0}-\sum_{1}^{\infty} b_{n}\right)+\left(x-\sum_{0}^{\infty} x_{n}\right) .
$$

Since $a+x$ is bounded, so is $x-\sum_{0}^{\infty} x_{n}$, and hence

$$
b_{0}-\sum_{1}^{\infty} b_{n}=\theta \text {. }
$$

For all $n, \phi^{-1}$ is defined on $x_{n}$, and

$$
\phi^{-1}\left(x_{n}\right) \leqq x_{n-1} \text {. }
$$

From this it follows that $\phi^{-n}$ is defined on $x_{n}$ and satisfies

$$
\phi^{-n}\left(x_{n}\right) \leqq \phi^{-n+1}\left(x_{n-1}\right) \leqq \cdots \leqq \phi^{-1}\left(x_{1}\right) \leqq x_{0},
$$

and hence that

$$
\phi^{-(n+1)}\left(x_{n}\right) \leqq \phi^{-(n-k+1)}\left(x_{n-k}\right) \leqq a_{0} \quad \text { for any } k<n \text {. }
$$


But from (8) we have

$$
\phi^{-1}\left(b_{n}\right)=x_{n-1}-\phi^{-1}\left(x_{n}\right),
$$

so we can define for $n \geqq 1$

$$
a_{n} \equiv \phi^{-(n+1)}\left(b_{n}\right)=\phi^{-n}\left(x_{n-1}\right)-\phi^{-(n+1)}\left(x_{n}\right) \leqq a_{0} .
$$

It follows from (19) and (16) that for $k>0$

$$
a_{n} a_{n+k} \leqq a_{n} \phi^{-(n+k)}\left(x_{n+k-1}\right) \leqq a_{n} \phi^{-(n+1)}\left(x_{n}\right)=\theta,
$$

and hence that the elements $a_{n}, n=1,2,3, \cdots$, are disjoint to each other. From this and from the fact that by definition $a_{n} \sim b_{n}\left(\phi^{n+1}\right)$, we have

$$
\sum_{1}^{\infty} a_{n} \sim \sum_{1}^{\infty} b_{n}=b_{0} .
$$

Since the element $x$ is disjoint to both sides of (21), this yields

$$
\sum_{1}^{\infty} a_{n}+x_{n} \sim b_{0}+x
$$

which with (12) gives

$$
\sum_{.1}^{\infty} a_{n}+x \sim a_{0}+x .
$$

But by hypothesis $a+x$ is bounded, and so

$$
a_{0}-\sum_{1}^{\infty} a_{n}=\theta \text {. }
$$

We now have, by combining (21), and (24),

$$
a_{0} \sim b_{0},
$$

which completes the proof of (4) and so of the required theorem.

4. Consequences of Theorem 3.2. We prove first the following statement.

4.1. Theorem. If $a, b$ are bounded and if $a^{\prime} \leqq a$ and $b^{\prime} \leqq b$, then $a \sim b$ and $a^{\prime} \sim b^{\prime}$ implies that $a-a^{\prime} \sim b-b^{\prime} ;$ i.e., for bounded elements, $\Phi$-equivalence is subtractive.

Proof. Let $a \sim b(\phi)$ and $a^{\prime} \sim b^{\prime}\left(\phi^{\prime}\right)$; then $a^{\prime} \sim b^{\prime} \sim \phi^{-1}\left(b^{\prime}\right) \leqq a$. Then by (3.2) $a-a^{\prime} \sim a-\phi^{-1}\left(b^{\prime}\right) \sim b-b^{\prime}(\phi)$. Therefore $a-a^{\prime} \sim b-b^{\prime}$.

4.11. TheOREM. If $\left(a_{n}\right)$ and $\left(b_{n}\right)$ are monotonic sequences of bounded elements of $M$ and if $a_{n} \sim b_{n}$ for all $n$, then $\lim a_{n} \sim \lim b_{n} ;$ i.e., the respective limits of monotonic sequences of bounded equivalent elemenis are equivalent.

Proof. Let us call these limits $a$ and $b$, respectively. Suppose $\left(a_{n}\right)$ and there- 
fore also $\left(b_{n}\right)$ are non-decreasing. Then $a=a_{1}+\left(a_{2}-a_{1}\right)+\left(a_{3}-a_{2}\right)+\cdots$, and $b=b_{1}+\left(b_{2}-b_{1}\right)+\left(b_{3}-b_{2}\right)+\cdots$. Then since $a_{n}$ and $b_{n}$ are bounded and $a_{n} \sim b_{n}$ for all $n$, by 4.1 we may sum corresponding sides and so get $a \sim b$. If the sequences are non-increasing, then from $4.1 a_{n}-a_{n-1} \sim b_{n}-b_{n-1}$. Therefore $a_{1}-a \sim b_{1}-b$. Since further $a_{1} \sim b_{1}$, by again applying 4.1 we get $a \sim b$.

4.12. TheOREM. The sum of any finite collection of bounded elements is bounded.

Proof. We shall prove 4.12 for the case of two bounded, disjoint elements $a_{1}$ and $a_{2}$, since the general case is readily reducible to this. Suppose then that under these conditions $a_{1}+a_{2}$ is unbounded. Then there exists an element $a^{\prime} \neq \theta$ contained in $a \equiv a_{1}+a_{2}$ such that $a-a^{\prime} \neq \theta$, and $a \sim a^{\prime}$. Then $a_{1}+a_{2}$ $\sim a_{1}^{\prime}+a_{2}^{\prime}$, where $a_{1}^{\prime}=\phi\left(a_{1}\right)$ and $a_{2}^{\prime}=\phi\left(a_{2}\right)$. From 3.2 we have

$$
\begin{aligned}
& a_{1}-a_{1} a_{1}^{\prime} \sim a_{1}^{\prime}-a_{1} a_{1}^{\prime}, \\
& a_{2}-a_{2} a_{2}^{\prime} \sim a_{2}^{\prime}-a_{2} a_{2}^{\prime} .
\end{aligned}
$$

But since $a_{1}^{\prime}-a_{1} a_{1}^{\prime}=a_{1}^{\prime} a_{2}$ and $a_{1}-a_{1} a_{1}^{\prime}=\left(a_{1}-a_{1} a^{\prime}\right)+a_{1} a_{2}^{\prime}$ we have

$$
\left(a_{1}-a_{1} a^{\prime}\right)+a_{1} a_{2}^{\prime} \sim a_{1}^{\prime} a_{2},
$$

and similarly

$$
\left(a_{2}-a_{2} a^{\prime}\right)+a_{2} a_{1}^{\prime} \sim a_{2}^{\prime} a_{1} .
$$

Now since the left-hand members of (3) and (4) are disjoint, we may substitute in (4) for $a_{1}^{\prime} a_{2}^{\prime}$ and we get

$$
\left(a_{2}-a_{2} a^{\prime}\right)+\left(a_{1}-a_{1} a^{\prime}\right)+a_{1} a_{2}^{\prime} \sim a_{1} a_{2}^{\prime} .
$$

But $a_{1} a_{2}^{\prime}$ is bounded and so

$$
\left(a_{2}-a_{2} a^{\prime}\right)+\left(a_{1}-a_{1} a^{\prime}\right)=a-a^{\prime}=\theta,
$$

contrary to hypothesis. Therefore $a$ is bounded.

It may be noted that the class $M$ of all bounded elements of $M$ is a finitely additive ideal (and a restricted $\sigma$-ideal) in $M$, and that the set of all limits of (sequences of) bounded elements is a $\sigma$-ideal in $M$.

5. Lattices $M$ derived from rings of sets. Let $m$ be a $\sigma$-ring of subsets of a fixed set $s ; n$, a $\sigma$-ideal in $m$; and $\Pi$ a class of univocal point transformations $\Pi_{\alpha}$, defined between pairs of sets in $m$ and leaving $m$ and $n$ invariant. Let $M$ be the residue class ring $m / n$, and suppose the isomorphisms on $M$ derived from the transformations of $\Pi$ (see 1.22) satisfy $(\beta)$. Suppose further that conditions $(\gamma)$ hold:

5.1. i. If $\mathrm{x}$ is any subset of $\mathrm{s}$ and if there exists a set $\mathrm{n} \in \mathrm{n}$ such that $\mathrm{x} \subseteq \mathrm{n}$, then $x \in n$.

ii. If $\mathrm{x} \subseteq s$, then there exists a set $\mathrm{x}^{*} \in m$ such that (a) $\mathrm{x} \subset \mathrm{x}^{*}$ and (b) if $x \subset a \in m$, then $x^{*}-x^{*} a \in n$. 
Let us denote the residue class of a set $\mathbf{x} \in m$ by $m(\mathbf{x})$ or by $x^{\prime}$. Then

5.11. If $\mathrm{x}_{1}^{*}$ and $\mathrm{x}_{2}^{*}$ satisfy $\mathrm{ii}$, then $x_{1}^{*}=x_{2}^{*}$.

5.12. If $\mathrm{x} \subseteq \mathrm{x}^{\prime}$, then $x^{*} \subseteq x^{\prime *}$.

5.13. For any $x \subseteq s$, there exists a set $x_{*}$ of $m$, namely, $x^{*}-\left(x^{*}-x\right)^{*}$, such that i. $x_{*} \subseteq x_{*}$;ii. if $x_{*}^{\prime} \subset x$, then $x_{*}^{\prime} \subseteq x_{*}$, and iii. if $x_{*}$ and $y_{*}$ satisfy $i$ and ii, then $x_{*}=y_{*}$.

5.14. A set $\mathrm{x} \subset \mathrm{s}$ is in $m$ if and only if $x^{*}=x_{*}$, in which case $x^{*}=x_{*}=x$.

5.15. If $\mathbf{x}_{n} \subset \mathbf{s}$, then for any $\mathbf{x}_{n}^{*}, \mathbf{x}_{n *}, m\left(\sum \mathbf{x}_{n}^{*}\right)=\sum m\left(\mathbf{x}_{n}^{*}\right) ; m\left[\left(\sum \mathbf{x}_{n}\right)_{*}\right]$ $\geqq m\left(\sum \mathbf{x}_{n *}\right) ; m\left[\left(\Pi \mathbf{x}_{n}\right)^{*}\right] \leqq m\left(\prod \mathbf{x}_{n}^{*}\right) ; m\left[\left(\prod \mathbf{x}_{n}\right)_{*}\right]=m\left(\prod \mathbf{x}_{n *}\right)$.

Since the proofs of these relations are all alike, we shall prove only the first. From the fact that $\left(\sum \mathbf{x}_{n}\right)^{*} \geqq \sum \mathbf{x}_{n}>\mathbf{x}$ for all $n, m\left(\mathbf{x}_{n}^{*}\right) \leqq m\left[\left(\sum \mathbf{x}_{n}\right)^{*}\right]$, and so $m\left[\left(\sum \mathbf{x}_{n}\right)^{*}\right] \geqq m\left[\sum \mathbf{x}_{n}^{*}\right]$. Similarly, $\mathbf{x}_{n}^{*} \geqq \mathbf{x}_{n}$ for all $n$, and so $\sum \mathbf{x}_{n}^{*} \geqq \sum \mathbf{x}_{n}$. Therefore $m\left(\sum \mathbf{x}_{n}^{*}\right)>m\left[\left(\sum \mathbf{x}_{n}\right)^{*}\right]$, and the relation is proved.

5.16. Lemma. A necessary and sufficient condition that $\mathrm{x} \in m$ is that for any $y \subseteq s,(x y)^{*}(y-x y)^{*} \in n$.

Proof. Suppose $\mathbf{x} \notin m$. Then for any $\mathbf{x}^{*}, \mathbf{x}^{*}-\mathbf{x}$ is not in $m$ and so not in $n$. Then from $\mathrm{i}$ of $(\gamma),\left(x^{*}-x\right)^{*}$ is not in $n$. Now let $y=x^{*}$. Then $(x y)^{*}(x-x y)^{*}$ $=\left(\mathbf{x}^{*}-\mathbf{x}\right)^{*} \notin n$. If $\mathbf{x} \in m$, then for any $\boldsymbol{y} \subseteq \mathbf{s},(x \boldsymbol{y})^{*} \leqq \mathbf{x}$, and so $m\left[(\mathbf{x y})^{*}\right] \leqq m(\boldsymbol{x})$. Also $y-x y<s-x<(s-x)^{*}-(s-x)^{*}(x y)^{*}$, which with ii of $(\gamma)$ completes the proof.

5.17. If $\pi$ is any transformation of $\Pi$ defined on a set $a \in m$, then for any $x \subset a$, we define $\pi(x)$ as the image of $x$ under $\pi$. Then since $\pi$ leaves $m$ and $n$ invariant, $\pi(\mathbf{a}) \in m$, and $\pi(\mathbf{a}) \supseteq \pi\left(\mathbf{x}^{*}\right) \supseteq \pi(\mathbf{x})$, so $m\left[\pi\left(\mathbf{x}^{*}\right)\right]>m\left[\pi^{*}(\mathbf{x})\right]$. But since $\pi^{*}(x) \supseteq \pi(x)$, it follows that $\pi^{-1}\left[\pi^{*}(x)\right] \supseteq x$. Therefore $\pi^{-1}\left[\pi^{*}(x)\right] \supseteq x^{*}$, whence $m\left[\pi\left(\mathbf{x}^{*}\right)\right]=m\left[\pi^{*}(\mathbf{x})\right]$. By a similar argument with reference to $\mathbf{x}$, we also have $m\left[\pi\left(\mathbf{x}_{*}\right)\right]=m\left[\pi_{*}(\mathbf{x})\right]$. That is, $m\left(\mathbf{x}^{*}\right) \sim m\left[\pi(\mathbf{x})^{*}\right]$, and $m(\mathbf{x})_{*} \sim m\left[\pi(\mathbf{x})_{*}\right]$.

\section{PART II}

6. Measuring systems in abstract sets. A set $s$ is said to have a measuring system $(m, n, \Phi)$ if $m$ is a $\sigma$-ring of subsets of $s ; n$, a $\sigma$-ideal in $m$ (notice that under these conditions $M \equiv m / n$ satisfies $(\alpha)$ of $\S 1$ ); and $\Phi$, a class of $\sigma$-isomorphisms defined on $M$ and satisfying $(\beta)(\S 2)$. If $(\gamma)$ of $\$ 5$ also holds, the system $(m, n, \Phi)$ is complete. Any set has a trivial measuring system, namely, (the totality of its subsets, the void set, the identity transformations on every subset). In euclidean space, the Lebesgue measurable sets, the null sets, and the transformations derived from direct sums of rigid motions form such a system.

Now let us consider a set $s$ with a given system $(m, n, \Phi)$. An isomorphism of $\Phi$ is said to be measure-preserving, and the elements $a, b, c, \cdots$, of $M$ to be measurable and of measure $M(a), M(b), M(c), \cdots,(2.2, \mathrm{i})$, respectively. Hencefor th these measures will be written $\mathfrak{a}$ or $\mathfrak{m}(a), \mathfrak{b}$ or $\mathfrak{m}(b), \mathfrak{c}$ or $\mathfrak{m}(c), \cdots$. In particular, the element $n$ is said to have null measure $\mathfrak{m}(n)=0$. The ele- 
ments $a$ and $b$ are of equal measure if and only if they correspond under some measure-preserving transformation, i.e., if and only if $a \sim b$. If the system $(m, n, \Phi)$ is complete, a set $a \in m$ is said to be measurable and of measure $\mathfrak{m}(m(\boldsymbol{a}))$, where $m(\boldsymbol{a})$ is the residue class of $\boldsymbol{a}$. For any $\mathbf{x} \subseteq \mathbf{s}$, the elements $x^{*}$ and $x_{*}(\S 5)$ are called a hull and a kernel of $\mathrm{x}$, respectively. Then $\mathfrak{m}\left(x^{*}\right)$ and $\mathfrak{m}\left(x_{*}\right)$, which will now be written $\mathfrak{x}^{*}$ and $\mathfrak{x}_{*}$, will be called the outer measure and the inner measure of $\mathbf{x}$.

7. Partial ordering of the measure-values. Addition. Let $\mathfrak{M}^{*}=(\mathfrak{a}, \mathfrak{b}, \mathfrak{c}, \cdots)$ be the totality of measures, and let $\mathfrak{M}$ be the subset of bounded measures (2.32). Henceforth $\mathfrak{a}<\infty$ shall mean $\mathfrak{a}$ is bounded.

7.1. Definition. If $\mathfrak{a}<\infty$, we define $\mathfrak{a}<\mathfrak{b}$ to mean that there exist corresponding elements $a$ and $b$ in $M$ such that $a<b$. It follows that $\mathfrak{a}<\mathfrak{b}$ is a (proper) partial ordering of $\mathfrak{M}$. The null element 0 of $\mathfrak{M}^{*}$ is the zero of the ordering.

7.11. Any monotonic increasing (decreasing) sequence $\left(\mathfrak{a}_{n}\right)$ has a supremum (an infimum) written $\lim \mathfrak{a}_{n}$. For suppose $\mathfrak{a}_{n}$ increasing, and let $a_{n}$ be a sequence of corresponding elements from $M$. For each $n$, there exists an $a_{n}^{\prime}<a_{n+1}$ such that $a_{n}^{\prime} \sim a_{n}$. Then $\mathrm{m}\left[\sum\left(a_{n+1}-a_{n}^{\prime}\right)\right]$ can be verified to be the desired supremum.

7.2. Definition. If $\left(\mathfrak{a}_{n}\right) \subset \mathfrak{M}^{*}$, and if there exists a sequence $\left(a_{n}\right)$ of disjoint corresponding elements in $M$, then $\left(\mathfrak{a}_{n}\right)$ is said to have a sum $\sum \mathfrak{a}_{n}$, namely, $\mathfrak{m}\left(\sum a_{n}\right)$. A binary sum will be written $\mathfrak{a}+\mathfrak{b}$.

It follows from the considerations of Part I that:

7.21. Whenever it exists, the sum is unique, and the operation of addition is commutative and associative, and $\sum \mathfrak{a}_{n}=\lim \sum_{1}^{n} a_{i}$.

7.22. If $\mathfrak{a}_{i}=\mathfrak{a}$ for all $i$, then we write $\sum_{1}^{n} \mathfrak{a}_{i}$ as $n \mathfrak{a}$. If either side of the following exists, so does the other; and $n \mathfrak{a}+m \mathfrak{a}=(n+m) \mathfrak{a} ; n(\mathfrak{a}+\mathfrak{b})=n \mathfrak{a}+n \mathfrak{b}$; $n(m \mathfrak{a})=m(n \mathfrak{a})=n m \mathfrak{a}$.

7.23. For any $\mathfrak{a} \in \mathfrak{M}^{*}, \mathfrak{a}+0$ exists and is equal to $\mathfrak{a}$; if $\mathfrak{a}<\infty$ then $\mathfrak{a}+\mathfrak{b}=\mathfrak{a}$ if and only if $\mathfrak{b}=0$.

7.24. Binary addition has a unique inverse for bounded elements; i.e., $\mathfrak{a}+\mathfrak{x}=\mathfrak{b}<\infty$ has a unique solution for fixed $\mathfrak{a}$ and $\mathfrak{b}$, denoted by $\mathfrak{b}-\mathfrak{a}$.

7.25. A necessary and sufficient condition that $\mathfrak{a}<\mathfrak{b}$ is that for some $\mathfrak{c}$, $\mathfrak{a}+\mathfrak{c}=\mathfrak{b}$ holds. If $\mathfrak{a}<\mathfrak{b}<\infty$ then there exists a maximum integer $n$ such that $n \mathfrak{a} \leqq \mathfrak{b}$.

7.26. If $\sum \mathfrak{m}\left(a_{n}\right)$ exists, then $\sum \mathfrak{m}\left(a_{n}\right) \geqq \mathfrak{m}\left(\sum a_{n}\right)$, and equality holds if and. only if the elements $a_{n}$ are disjoint, in which case $\sum \mathfrak{m}\left(a_{n}\right)$ does exist.

\section{Algebraic properties of $\mathfrak{M}^{*}$.}

8.1. We now sec that any measuring system $(m, n, \Phi)$ gives rise to a set $\mathfrak{M}^{*}$ of measure-values with a commutative and associative operation of addition defined for certain denumerable sets of elements of $\mathfrak{M}^{*}$, in terms of which a'partial ordering of the bounded elements can be derived. Then 
i. There exists an element 0 such that $\mathfrak{a} \neq 0$ implies that $\mathfrak{a}>0$ and $\mathfrak{a}+0=\mathfrak{a}$.

ii. If $a<\infty$, and $a<b$, then $\mathrm{m}(a)<\mathfrak{m}(b)$.

iii. $\sum \mathfrak{m}\left(a_{n}\right) \geqq \mathfrak{m}\left(\sum a_{n}\right)$ and equality holds if and only if the elements $a_{n}$ are disjoint.

If, further, the measure defined is complete, then to any $x \subseteq s$ there corresponds an outer and an inner measure $\mathfrak{r}^{*}$ and $\mathfrak{x}_{*}$, respectively, and

iv. If $x^{*}<\infty$, and $\mathrm{x}<\mathrm{y}$, then $\mathrm{x}^{*} \leqq \mathrm{y}^{*}$.

v. If $\left(x_{n}\right)$ is a sequence of sets such that $\sum x_{n}{ }^{*}$ exists, then $\mathfrak{m}\left(\sum x_{n}\right)^{*} \leqq \sum \mathfrak{x}_{n}{ }^{*}$ if $\left(\sum \mathbf{x}_{n}\right)^{*}<\infty$.

vi. The set $\mathrm{x}$ is measurable if and only if the equivalent conditions a and $\mathrm{b}$ (the Carathéodory condition) hold:

a. $x^{*}=x_{*}$, in which case $x^{*}=x_{*}=x$, and

b. for any $y \subseteq s, \mathfrak{m}(x y)^{*}+\mathfrak{m}(y-x y)^{*}=y^{*}\left[=\mathfrak{m}\left((x y)^{*} \cdot(y-x y)^{*}\right)\right]$.

9. Ordered sets of measure-values. In the remainder of Part II it is assumed that the system $(m, n, \Phi)$ satisfies condition $(\delta)$.

( $\delta$ ) For any $a, b$ of $M$ either there exists $a b^{\prime} \leqq b$ such that $a \sim b^{\prime}$ or there exists an $a^{\prime}<a$ such that $a^{\prime} \sim b$.

9.1. A necessary and sufficient condition that $(\delta)$ hold is that for any two elements $\mathfrak{a}$ and $\mathfrak{b}$ of $\mathfrak{M}^{*}$ either there exists an $\mathfrak{a}^{\prime}$ such that $\mathfrak{a}+\mathfrak{a}^{\prime}=\mathfrak{b}$ or $a \mathfrak{b}^{\prime}$ such that $\mathfrak{b}+\mathfrak{b}^{\prime}=\mathfrak{a}$.

We shall show that $(\delta)$ is a sufficient as well as a trivially necessary condition that $M$ be order and addition isomorphic to a set of positive numbers.

9.2. For any bounded measures $\mathfrak{a}$ and $\mathfrak{b}, \mathfrak{a} \nless \mathfrak{b}$ implies that $\mathfrak{a} \geqq \mathfrak{b}$; i.e., $\mathfrak{M}$ is linearly ordered, and either $\mathfrak{b}-\mathfrak{a}$ or $\mathfrak{a}-\mathfrak{b}$ exists.

9.21. From 9.2, one and only one of the following occurs: a. $\mathfrak{M}$ contains the single element $0 ; b$. there exists an element $\mathfrak{a}$ of $\mathfrak{M}$ such that $a \neq 0$, and if $\mathfrak{a}^{\prime}<\mathfrak{a}$, then $\mathfrak{a}^{\prime}=0$; . for any $\mathfrak{a}>0$ there exists an $\mathfrak{a}^{\prime}$ such that $0<\mathfrak{a}^{\prime}<\mathfrak{a}$. In the last case, we may assume that $2 \mathfrak{a}^{\prime} \leqq \mathfrak{a}$, for we can take the lesser of $\mathfrak{a}^{\prime}$ and $\mathfrak{a}-\mathfrak{a}^{\prime}$.

9.22. If $\mathfrak{a}_{n}, \mathfrak{a}$ are bounded, and $\mathfrak{a}_{n} \leqq \mathfrak{a}_{n+1}\left(\mathfrak{a}_{n} \geqq \mathfrak{a}_{n+1}\right)$, then $\mathfrak{a}<\lim \mathfrak{a}_{n}\left(\mathfrak{a}>\lim \mathfrak{a}_{n}\right)$ implies that there exists a $k$ such that $\mathfrak{a} \leqq \mathfrak{a}_{k}\left(\mathfrak{a} \geqq \mathfrak{a}_{k}\right)$.

9.3. i. For any $\mathfrak{a}, \mathfrak{b}$ in $\mathfrak{M}$, there is a unique integer $n$ such that $\mathfrak{b}=n \mathfrak{a}+\mathfrak{c}$, $\mathfrak{c}<\mathfrak{a}$. ii. If $\mathfrak{a}$ and $\mathfrak{b}$ are bounded, then for every integer $n$ such that $n \mathfrak{a}$ and $n \mathfrak{b}$ exist, $n \mathfrak{a}$ and $n \mathfrak{b}$ stand in the same relative order as do $\mathfrak{a}$ and $\mathfrak{b}$.

9.4. There exists an element $s$ of $M^{*}$ such that $\mathfrak{a}<\infty$ implies that $\mathfrak{a} \leqq \mathbb{8}$. If $z \nless \infty$, the sum of any two bounded elements always exists; if $z<\infty, \mathfrak{a}+\mathfrak{b}$ exists if and only if the equivalent conditions $\mathfrak{a}<\boldsymbol{b}-\mathfrak{b}$ and $\mathfrak{b}<\boldsymbol{b}-\mathfrak{a}$ hold. In this latter case, the element $s$ is unique; and for any $a$ of $M, a \leqq s$.

9.5. If $\mathfrak{M}$ satisfies $b$ of 9.21 , that is, if there is a least element $a_{1}>0$, then it follows from 9.3 and 9.4 that the elements of $\mathfrak{M}$ are of the form $n \mathfrak{a}_{1}$, and that according as 8 is unbounded or bounded and equal to $k a_{1}, n$ will assume all positive integral values or all those not greater that $k$.

9.6. Lemma. If $\mathfrak{M}$ satisfies $\mathrm{c}$ of 9.21 , i.e., $\mathfrak{M}$ has no minimal non-null ele- 
ments, and, if each element of $\mathfrak{M}$ is contained in one or the other, but not both of two non-void subclasses $\mathfrak{M}^{\prime}$ and $\mathfrak{M}^{\prime \prime}$ and if every element of $\mathfrak{M}^{\prime \prime}$ is greater than every element of $\mathfrak{M}^{\prime}$, then either there is a least element of $\mathfrak{M}^{\prime \prime}$ and no greatest in $\mathfrak{M}^{\prime}$, or there is a greatest element in $\mathfrak{M}^{\prime}$ and no least in $\mathfrak{M}^{\prime \prime}$.

Proof. Since neither $\mathfrak{M}^{\prime}$ nor $\mathfrak{M}^{\prime \prime}$ is void there exist elements $\mathfrak{a}_{1}^{\prime}$ in $\mathfrak{M}^{\prime}$ and $\mathfrak{a}_{1}^{\prime \prime}$ in $\mathfrak{M}^{\prime \prime}$ with $\mathfrak{a}_{1}^{\prime}<\mathfrak{a}_{1}^{\prime \prime}$. From c, there is a third element between the two, so both cannot be the extreme elements of their respective classes. If either one is, the lemma is proved; if not, since there is some $\mathfrak{c}_{1}>0$ such that $2 \mathfrak{c}_{1} \leqq \mathfrak{a}_{1}^{\prime \prime}-\mathfrak{a}_{1}^{\prime}$, there is a maximum integer $n_{1}$ such that $\mathfrak{a}_{1}^{\prime}+n_{1} \mathfrak{c}_{1}<\mathfrak{M}^{\prime}$, and a maximum integer $n_{1}^{\prime} \geqq n_{1}$ such that $\mathfrak{a}_{1}^{\prime}+n_{1}^{\prime} \mathfrak{c}_{1} \leqq \mathfrak{a}_{1}^{\prime \prime}$. Let $\mathfrak{a}_{2}^{\prime}=\mathfrak{a}_{1}^{\prime}+n_{1} \mathfrak{c}_{1}$ and let $\mathfrak{a}_{2}^{\prime \prime}$ be either $\mathfrak{a}_{1}^{\prime}+\left(n_{1}^{\prime}+1\right) \mathfrak{c}_{1}$ or $\mathfrak{a}_{1}^{\prime \prime}$, according as $n_{1}^{\prime}>n_{1}$ or $n_{1}^{\prime}=n_{1}$. Then $\mathfrak{a}_{2}^{\prime} \in \mathfrak{M}^{\prime}, \mathfrak{a}_{2}^{\prime \prime} \in \mathfrak{M}^{\prime \prime}, \mathfrak{a}_{1}^{\prime} \leqq \mathfrak{a}_{2}^{\prime}<\mathfrak{a}_{2}^{\prime \prime} \leqq \mathfrak{a}_{1}^{\prime \prime}$, and $2\left(\mathfrak{a}_{2}^{\prime \prime}-\mathfrak{a}_{2}^{\prime}\right) \leqq\left(\mathfrak{a}_{1}^{\prime \prime}-\mathfrak{a}_{1}^{\prime}\right)$. Let $\mathfrak{c}_{2}$ be the minimum of $\mathfrak{a}_{1}^{\prime \prime}-n_{1}^{\prime} \mathfrak{c}_{1}$ and $\mathfrak{c}_{1}-\left(\mathfrak{a}_{1}^{\prime \prime}-n_{1}^{\prime} \mathfrak{c}_{1}\right)$. Then $2 \mathfrak{c}_{2} \leqq \mathfrak{c}_{1}$ and hence $4 \mathfrak{c}_{2} \leqq \mathfrak{a}_{1}^{\prime \prime}-\mathfrak{a}_{1}^{\prime}$. Either if $\mathfrak{a}_{2}^{\prime}$ is the maximum of its class, or if $\mathfrak{a}_{2}^{\prime \prime}$ is the minimum of its class, the assertion holds. If not, we define $\mathfrak{a}_{3}^{\prime}$ and $\mathfrak{a}_{3}^{\prime \prime}$ just as in the preceding case. By proceeding in this way, we arrive at a pair of sequences $\left(\mathfrak{a}_{n}^{\prime}\right)$ and $\left(\mathfrak{a}_{n}^{\prime \prime}\right)$ of elements of $\mathfrak{M}^{\prime}$ and $\mathfrak{M}^{\prime \prime}$, respectively, such that $\mathfrak{a}_{n}^{\prime} \leqq \mathfrak{a}_{n+1}^{\prime} \leqq \mathfrak{a}_{n+1}^{\prime \prime}$ $\leqq \mathfrak{a}_{n}^{\prime \prime}$ and $2^{n-1}\left(\mathfrak{a}_{n}^{\prime \prime}-\mathfrak{a}_{n}^{\prime}\right) \leqq \mathfrak{a}_{1}^{\prime \prime}-\mathfrak{a}_{1}^{\prime}$. The sequences are monotonic and contained in $M$, so their respective limits $\mathfrak{a}^{\prime}$ and $\mathfrak{a}^{\prime \prime}$ exist and are bounded. Also, since for any $\mathfrak{b}^{\prime} \in \mathfrak{M}^{\prime}$ and for any integer $m, \mathfrak{b}^{\prime}<\mathfrak{a}_{m}{ }^{\prime \prime}$, it follows from 7.11 that $\mathfrak{b}^{\prime} \leqq \mathfrak{a}^{\prime \prime}=\lim \mathfrak{a}_{n}^{\prime \prime}$. In particular this relation holds for all elements of $\left(\mathfrak{a}_{n}^{\prime}\right)$, and so $\mathfrak{a}^{\prime}=\lim \mathfrak{a}_{n}{ }^{\prime} \leqq \mathfrak{a}^{\prime \prime}$. Further since $\mathfrak{a}_{n}^{\prime} \leqq \mathfrak{a}^{\prime} \leqq \mathfrak{a}^{\prime \prime} \leqq \mathfrak{a}_{n}^{\prime \prime}$ for all $n$, it follows that $\mathfrak{a}^{\prime \prime}-\mathfrak{a}^{\prime} \leqq \mathfrak{a}_{n}^{\prime \prime}-\mathfrak{a}_{n}^{\prime} \leqq 2^{n-1}\left(\mathfrak{a}_{1}^{\prime \prime}-\mathfrak{a}_{1}^{\prime}\right)$ and hence that $\mathfrak{a}^{\prime \prime}-\mathfrak{a}^{\prime}=\theta$, i.e., $\mathfrak{a}^{\prime}=\mathfrak{a}^{\prime \prime}=\mathfrak{a}$. From the fact that $\mathfrak{a}$ is the limit of a sequence from $\mathfrak{M}^{\prime}$ and of one from $\mathfrak{M}^{\prime \prime}$ it follows that $\mathfrak{a}$ is the greatest or the least element of its class according as it is in $\mathfrak{M}^{\prime}$ or in $\mathfrak{M}^{\prime \prime}$, and that the class to which a does not belong has no extreme element.

\section{Isomorphisms of $\mathfrak{M}$ onto sets of positive numbers.}

10.1. ThEOREM. Under condition ( $\delta$ ) the set $\mathfrak{M}$ of bounded measure-values is isomorphic to a subset $\mathbf{F}$ of the set of non-negative numbers; i.e., there exists a one-to-one mapping $f(\mathfrak{a})$ of $\mathfrak{M}$ onto $\Gamma$ such that whenever $\sum \mathfrak{a}_{n}$ exists and is bounded then $\sum f\left(\mathfrak{a}_{n}\right)$ converges to $f\left(\sum \mathfrak{a}_{n}\right)$, and whenever $\sum \mathfrak{a}_{n}$ exists and is unbounded, then $\sum f\left(\mathfrak{a}_{n}\right)$ diverges to infinity. The mapping $f$ is completely determined up to a multiplicative constant; that is, if $f^{\prime}$ is any other mapping of $M$ onto $\Gamma$ with the above property, then there exists a constant $\xi$ such that for all $a<\infty, f(\mathfrak{a})=\xi f^{\prime}(\mathfrak{a})$. According as conditions $\mathrm{a}, \mathrm{b}$, or $\mathrm{c}$, of 9.21 hold, the set $\Gamma$ will consist of $\mathrm{a}$. the number zero, b. the set $n f\left(a_{1}\right)$, where $n=1,2,3, \cdots$ or $1,2, \cdots, k$, or $c$. the interval $0 \leqq \xi \leqq f(8)$, or $0 \leqq \xi \leqq \infty$, according as the element 8 of 9.4 is bounded or unbounded.

The theorem follows immediately for cases a and b. In case $c$, we shall use the following lemma: 
Lemma. To every ordered pair $(\mathfrak{a}, \mathfrak{b})$ of bounded elements different from 0 , there corresponds a unique positive finite number $\mu(\mathfrak{a}, \mathfrak{b})$ such that

i. if $\sum \mathfrak{a}_{n}$ exists, then $\mu\left(\sum \mathfrak{a}_{n}, \mathfrak{b}\right)=\sum \mu\left(\mathfrak{a}_{n}, \mathfrak{b}\right)$,

ii. $\mu(\mathfrak{a}, \mathfrak{a})=1$.

Proof. For any $\mathfrak{a} \neq 0$, the set $\mathfrak{M}^{\prime} \subseteq \mathfrak{M}$ of elements $\mathfrak{a}^{\prime}$ such that $2 \mathfrak{a}^{\prime} \leqq \mathfrak{a}$, and the set $\mathfrak{M}^{\prime \prime} \subseteq \mathfrak{M}$ of elements $\mathfrak{a}^{\prime \prime}$ such that $2 \mathfrak{a}^{\prime \prime}>\mathfrak{a}$, satisfy the hypotheses of 9.6. From this it follows that there exists an element $\mathfrak{a}^{1}$ such that $2 \mathfrak{a}^{1}=\mathfrak{a}$, and such that for every integer $n$ an $\mathfrak{a}^{n}$ exists such that $2^{n} \mathfrak{a}^{n}=\mathfrak{a}$. Then

$$
\begin{aligned}
\mathfrak{a}^{n} & =2^{k} \mathfrak{a}^{n+k}, \\
\sum_{i=1}^{p} \mathfrak{a}^{n_{i}} & =\left(\sum_{1}^{p} 2^{n_{i}-m}\right) \mathfrak{a}^{m} \quad \text { if } n_{i}>m \text { for } i=1,2, \cdots, p .
\end{aligned}
$$

Further if $\mathfrak{a}^{\prime}>0$, there exists an $n$ such that $\mathfrak{a}^{\prime}>\mathfrak{a}^{n}$. For by 9.4 there exists a $k$ such that $\mathfrak{a}=k \mathfrak{a}^{\prime}+\mathfrak{a}^{\prime \prime}$, where $\mathfrak{a}^{\prime \prime}<\mathfrak{a}^{\prime}$, and we may take $n=k+1$. More generally,

$$
\text { if } \mathfrak{a}^{\prime}>\mathfrak{a}^{\prime \prime}, \text { there exists an } n \text { for which } \mathfrak{a}^{\prime}<\mathfrak{a}^{\prime \prime}+\mathfrak{a}^{n} .
$$

For every pair of elements $\mathfrak{a}$ and $\mathfrak{b}$ there exists an integer $\mu_{n}(\mathfrak{a}, \mathfrak{b})$ such that $\mathfrak{a}=\mu_{n} \mathfrak{b}^{n}+\mathfrak{c}_{n}$, where $\mathfrak{c}_{n}<\mathfrak{b}^{n}$. From the previous, it follows that the $\mu_{n}$ satisfy

$$
2 \mu_{n} \leqq \mu_{n-1} \leqq 2\left(\mu_{n}-1\right) ;
$$

(5) if $\mathfrak{c}>\mathfrak{a}$, there exists a $k$ such that for all $p>0, \mu_{k+p}(\mathfrak{c}, \mathfrak{b})>\mu_{k+p}(\mathfrak{a}, \mathfrak{b})$;

$$
\begin{gathered}
\mu_{k}\left(\sum_{i=1}^{p} \mathfrak{a}^{n_{i}}, \mathfrak{a}\right)=\sum_{1}^{p} \mu_{k}\left(\mathfrak{a}^{n_{i}}, \mathfrak{a}\right) \quad \text { for } n_{i}>k \text { for } i=1,2, \cdots, p ; \\
\mu_{r}\left(\mathfrak{b}+k \mathfrak{a}^{r}, \mathfrak{a}\right)=\mu_{r}(\mathfrak{b}, \mathfrak{a})+k / 2^{r}, \quad \text { for any } \mathfrak{b} .
\end{gathered}
$$

From (4) the sequence of numbers $\mu_{n}(\mathfrak{b}, \mathfrak{a})$ is monotonically increasing and bounded and therefore approaches a unique limit $\mu(\mathfrak{b}, \mathfrak{a})$. We have at once that

$$
\begin{gathered}
\mu(0, \mathfrak{a})=0 ; \quad \mu\left(\mathfrak{a}_{n}, \mathfrak{a}\right)=1 / 2^{n} \\
\text { if } \mathfrak{c}<\mathfrak{b}, \text { then } \mu(\mathfrak{c}, \mathfrak{a})<\mu(\mathfrak{b}, \mathfrak{a}) \\
\mu\left(\mathfrak{c}+k \mathfrak{a}^{r}\right)=\mu(\mathfrak{c})+k / 2^{r}
\end{gathered}
$$

Also for any finite $p, \mu\left(\sum_{1}^{p} \mathfrak{a}_{i}, \mathfrak{a}\right)=\sum_{1}^{p} \mu\left(\mathfrak{a}_{i}, \mathfrak{a}\right)$. For $\sum_{1}^{p} \mathfrak{a}_{i}=\sum_{1}^{p} \mu_{k}\left(\mathfrak{a}^{\prime}, \mathfrak{a}\right) \mathfrak{a}^{k}$ $+\sum_{i=1}^{p} \mathfrak{a}_{\mathfrak{k}, \mathfrak{i}}^{\prime}$, where $\mathfrak{a}_{\mathfrak{k}, i}^{\prime}<\mathfrak{a}^{k}$. It can be seen that $\mu_{k}\left(\sum_{i=1}^{p} \mathfrak{a}_{i}, \mathfrak{a}\right)<p$; then applying (10), dividing by $2^{k}$ and passing to the limit give the desired result. Now suppose that $\mathfrak{a}^{*}=\sum_{1}^{\infty} \mathfrak{a}_{i}$ exists. If $\mathfrak{a}^{*}<\infty$, then since for every $n \mu_{n}\left(\mathfrak{a}^{*}, \mathfrak{a}\right) \mathfrak{a}^{n} \leqq \mathfrak{a}^{*}$ it follows that there is a $p_{n}$ for which $\mu_{n}\left(\mathfrak{a}^{*}, \mathfrak{a}\right) \mathfrak{a}^{n} \leqq \sum_{1}^{p} \mathfrak{a}_{i \cdot}$. Then $\mu_{n}\left(\mathfrak{a}^{*}, \mathfrak{a}\right) / 2^{n}$ $=\mu\left[\mu_{n}\left(\mathfrak{a}^{*}, \mathfrak{a}\right) \mathfrak{a}^{n}\right] \leqq \mu\left(\sum_{\mathfrak{i}=1}^{p} \mathfrak{a}_{i}, \mathfrak{a}\right)<\mu\left(\mathfrak{a}^{n}, \mathfrak{a}\right)$, or $\mu\left(\mathfrak{a}^{*}, \mathfrak{a}\right)-\mu\left(\sum_{1}^{p} \mathfrak{a}_{i}, \mathfrak{a}\right)<1 / 2^{n}$. If $\sum_{1}^{\infty} \mathfrak{a}_{i} \nless \infty$, then for any $n, n \mathfrak{a}<\sum_{1}^{\infty} \mathfrak{a}_{i}$, whence $\mu\left(\sum \mathfrak{a}_{\mathfrak{i}}, \mathfrak{a}\right)>\mu(n \mathfrak{a}, \mathfrak{a}) \geqq n$. 
Complete additivity of $\mu(\mathfrak{b}, \mathfrak{a})$ follows from these last two relations and the finite additivity; i.e.,

$$
\mu\left(\sum \mathfrak{a}_{i}, \mathfrak{a}\right)=\sum \mu\left(\mathfrak{a}_{i}, \mathfrak{a}\right) .
$$

Relations $\mathrm{i}$ and ii of the lemma are now proved, and it remains to show for each ordered pair $\mathfrak{b}, \mathfrak{a}$ that $\mu(\mathfrak{b}, \mathfrak{a})$ is unique. But any $\mu^{\prime}(\mathfrak{b}, \mathfrak{a})$ satisfying $i$ and ii can be seen to be such that

$$
k \mu^{\prime}\left(\mathfrak{a}^{n}, \mathfrak{a}\right)=k / 2^{n}=\mu\left(\mathfrak{a}^{n}, \mathfrak{a}\right),
$$

for all $k$ and $n$;

and so

$$
\mu^{\prime}(\mathfrak{b}, \mathfrak{a})=\mu^{\prime}\left(\lim \mu_{n}(\mathfrak{b}, \mathfrak{a}) \mathfrak{a}^{n}\right)=\lim \mu^{\prime}\left(\mu_{n}(\mathfrak{b}, \mathfrak{a}) \mathfrak{a}^{n}\right)=\lim \mu_{n} / 2^{n}=m(\mathfrak{b}, \mathfrak{a}) .
$$

We can also verify by a limiting process that if none of $\mathfrak{a}, \mathfrak{b}, \mathfrak{c}$ is 0 , then

$$
\mu(\mathfrak{a}, \mathfrak{b})=\mu(\mathfrak{a}, \mathfrak{c}) \mu(\mathfrak{c}, \mathfrak{b}) .
$$

Then the isomorphism required in 10.1 can be given by $f(\mathfrak{a})=\mu\left(\mathfrak{a}, \mathfrak{a}_{1}\right)$ for any $\mathfrak{a}_{1}>0$. From (14), $f(\mathfrak{a}) / f(\mathfrak{b})=\mu\left(\mathfrak{a}, \mathfrak{a}_{1}\right) / \mu\left(\mathfrak{b}, \mathfrak{a}_{1}\right)=\mu(\mathfrak{a}, \mathfrak{b})$, which is independent of $f$ and of $\mathfrak{a}_{1}$. But if $g(\mathfrak{a})$ is any mapping whatever satisfying the conditions of 10.1 , then the ratio $g(\mathfrak{a}) / g(\mathfrak{b})$ satisfies the conditions of the lemma, and so is equal to $\mu(\mathfrak{a}, \mathfrak{b})$. Therefore $g(\mathfrak{a})=g\left(\mathfrak{a}_{1}\right) \mu\left(\mathfrak{a}, \mathfrak{a}_{1}\right)$, and $g(\mathfrak{a}) / f(\mathfrak{a})=g\left(\mathfrak{a}_{1}\right)$, a constant.

Let $\xi$ be any positive finite number less than $\mu(\mathfrak{z}, \mathfrak{a})$, where $\mathfrak{a} \neq 0$, and $\xi$ satisfies 9.4. Then $\xi$ can be expressed uniquely in the form $\zeta+\xi / 2^{k}$ where $\zeta$ is an integer, $\xi_{k}=0$ or 1 and an infinite set of the $\xi_{k}$ are zero. It follows from 9.3 and relation (11) that all the sums $\xi_{k} \mathfrak{a}^{k}$ exist and that $\mu\left(\sum \xi_{k}\right) \mathfrak{a}=\xi$. Therefore the range of any isomorphism, which must be of the form $f(\mathfrak{b})=\mu(\mathfrak{b}, \mathfrak{a})$, is a closed finite or infinite interval with left end point zero. This completes the proof of 10.1 .

10.2. Let $s^{\prime}$ and $s^{\prime \prime}$ be any two unbounded elements of $M$, if such exist, which are the respective limits of monotonic increasing sequences $\left(s_{n}^{\prime}\right)$ and $\left(s_{n}^{\prime \prime}\right)$ of bounded elements. Then there exist subsequences $\left(s_{n_{i}}{ }^{\prime}\right)$ and $\left(s_{n_{i}}{ }^{\prime \prime}\right)$ such that $s_{n_{i}} \leqq s_{n_{i}}^{\prime \prime}<s_{n_{i+1}}^{\prime}$. It follows that $\bar{z}^{\prime}=\bar{g}^{\prime \prime}$, that is, all unbounded limits of bounded elements of $M$ are of equal measure, which we denote by $+\infty$. If the system $(m, n, \Phi)$ is such that

$(\epsilon)$ every element of $M$ is the limit of a sequence of bounded elements, then the range of measure-values in $\mathfrak{M}^{*}$ is $0 \leqq \xi \leqq+\infty$.

11. Isomorphisms of $\mathfrak{M}$ onto the infinite half-interval. Conclusion. We have shown that for any set $s$, any system $(m, n, \Phi)$ satisfying $(\alpha)$ and $(\beta)$ leads to a set $\mathfrak{M}^{*}$ of measures, the bounded subset $\mathfrak{M}$ of which satisfies conditions which may be regarded as a generalization of the Lebesgue conditions on a numerical measure. If in addition $(\delta)$ holds, the set $\mathfrak{M}^{*}$ of limits of bounded measures is isomorphic to either a set of successive non-negative integers or to a closed interval with left end points zero. If $(\epsilon)$ holds, i.e., if 
$\mathfrak{M}_{*}=\mathfrak{M}^{*}$, the measure determined by $(m, n, \Phi)$ can b€ represented by a function $f(a)$ which is unique up to a multiplicative constant, assumes the values $0 \leqq \xi \leqq \infty$, and is such that $f(a)=0$ if and only if $a=\theta ; f(a)=f(b)$ if and only if $a$ and $b$ correspond under some measure-preserving transformation, and $f(a)<\infty$ if and only if $a<\infty$. It may be pointed out that no reference is made to any topological or metric properties of the set $\mathbf{s}$.

It is not necessarily true that every numerical measure can be derived by the procedure above, for this would require the existence of a measure-preserving transformation between any two sets of equal measure. However, it is shown in Part III that this is true for many important measures. The general case can be treated by introducing into $M$ an equivalence relation, equality of measure, which is countably disjointly additive, hereditary in the sense of 2.2 , and for bounded elements is also subtractive and preserved by taking limits of monotonic sequences. It can easily be verified that the entire theory of Part II would hold in this case. Further if $M$ has a countable basis, a family of isomorphisms can be defined which generate the given congruence, which reduces this case to the previous one.

\section{PART III}

12. Lattices satisfying $(\zeta)$. Bases. Let $M$ be a lattice satisfying $(\alpha)$ of $\S 1$ and $|a|$ be a functional defined on $M$ and satisfying $(\zeta)$ :

ia. $0 \leqq|a| \leqq \infty$, for any $a$ of $M$, and $|a|=0$ if and only if $a=\theta$;

ib. if $|a|=\infty$, then there exists a sequence of elements $a_{n}$ such that $|a|<\infty$, and $\sum a_{n}=a$;

ii. $\left|\sum a_{n}\right|=\sum\left|a_{n}\right|$, for any sequence of disjoint $a_{n}$;

iii. for any $a \neq \theta$ there is an $a^{\prime}<a$ such that $0 \leqq|a| \leqq \infty$;

iv. for any a of $M$, there is a countable set of elements $a_{n}$ of $M$ such that for any $a^{\prime}<a$, and for any $\epsilon>0$, some subsequence $\left(a_{m}\right)$ covers $a^{\prime}$ and $\left|\sum a_{m}-a^{\prime}\right|<\epsilon$.

The object of the next sections is to prove under conditions $(\zeta)$ that if $|a|=\left|a^{\prime}\right|$ then there exists a $\sigma$-isomorphism $\phi$ of $I(a)$ on to $I\left(a^{\prime}\right)$ (see 1.1) such that for any $b<a,|\phi(b)|=|b|$, which in turn leads to the result that the $|a|$ can be determined up to a constant multiple by a set of isomorphisms satisfyig $(\beta)$.

Henceforth a family of elements $a_{n}$ satisfying iv of $(\zeta)$ will be called a basis of $a$, and any element of a basis a base element.

12.1. From the fact that if each element of a basis can be expressed as the sum of elements of a family $\left(a_{n}^{\prime}\right)$ then $\left(a_{n}^{\prime}\right)$ is also a basis, it follows that $a$ has a basis of the following form:

Let $\left(b_{n}\right)$ be any basis of $a$. Then let $a_{n}$ be the $n$th element of the form $b_{k}-\sum_{1}^{k-1} b_{i}$ which is different from $\theta$; let $a_{n, 0}$ be the first element of the form $a_{n} b_{k+i}$, where $a_{n}$ is derived from $b_{k}$ such that $\theta<a_{n} b_{k+i}<a_{n}$; and let $a_{n, 1}=a_{n}-a_{n, 0}$. Then if $a_{\nu}$ is defined for every sequence $\nu=\left(n, \epsilon_{1}, \cdots, \epsilon_{k}\right)$, $\epsilon_{i}= \pm 1$, as some product $\epsilon_{1} b_{1} \cdots \epsilon_{k} b_{k}$ where $-b=a-b$, then $a_{\nu, 0}$ is given 
by $\epsilon_{1} b_{1} \cdots \epsilon_{k} b_{k} b_{m}$, where $m$ is the least value for which $0<\epsilon_{1} b_{1} \cdots \epsilon_{k} b_{k} b_{m}$ $<\epsilon_{1} b_{1} \cdots \epsilon_{k} b_{k}$, and $a_{\nu, 1}=a_{\nu}-a_{\nu, 0}$. It follows at once that every $b_{n}$ is the sum of a finite set of the $a_{\nu}$. Therefore $\left(a_{\nu}\right)$ is a basis. Let $n(\nu)$ be the number of terms in the sequence $\nu$, and $\Gamma_{k}$ the totality of sequences $\nu$ such that $n(\nu)=k$. We define $\gamma<\nu$, where $\gamma=\left(n_{1}, \epsilon_{1}, \cdots, \epsilon_{k}\right)$ and $\nu=\left(n^{\prime}, \epsilon_{1}^{\prime}, \cdots, \epsilon_{k}^{\prime}\right)$ if $k>k^{\prime}$, and $\left(n, \epsilon_{1}, \cdots, \epsilon_{k}\right) \equiv\left(n^{\prime}, \epsilon_{1}^{\prime}, \cdots, \epsilon_{k}^{\prime}\right)$.
12.2. Then
i. $\sum a_{\gamma}=a, \gamma \in \Gamma_{n}$, for any fixed $n$;
ii. $a_{\nu} a_{\gamma}=\theta$ if $n(\nu)=n(\gamma)$;
iii. $a_{\nu}<a_{\gamma}$ if and only if $\nu<\gamma$;
iv. $a_{\gamma}=\sum a_{\nu}, \nu<\gamma, n(\nu)=n_{0}<n(\gamma)$.

Now let $\left(a_{\nu}\right)$ be any basis satisfying 12.2. It follows that:

12.3. If $\left(a_{v_{i}}\right)$ is any finite set of distinct base elements, then (a) $\prod a_{\nu_{i}}$ is equal either to $\theta$ or to the (necessarily unique) element of least subscript $\nu_{k}$, according as the sequence is or is not nested; (b) if $\nu_{i}<\nu$ then $a_{\nu}-\sum a_{\nu_{i}}$ is the sum of a finite set of disjoint base elements of subscripts $\nu_{k}^{\prime}$ such that $n\left(\nu_{i}^{\prime}\right)=n\left(\nu_{k}\right)$; (c) any countable sum of base elements can be expressed as the sum of disjoint base elements. (For $\sum a_{v_{i}}=\sum\left(a_{v_{i}}-\sum_{j<i} a_{v_{j}} a_{v_{i}}\right)$, where the terms of the right side are disjoint, and from (b) each consists of a finite sum of disjoint elements.)

Further, if $\left(a_{\nu}\right)$ and $\left(b_{v}\right)$ are bases of $a$ and $b$, respectively, which satisfy 12.2:

12.4. For any finite sequence $\left(\nu_{i}\right)$ of which we suppose $\nu_{k}$ the least (a) either both $\prod a_{\nu_{i}}=\prod b_{\nu_{i}}=\theta$, or $\prod a_{\nu_{i}}=a_{\nu_{k}}$ and $\prod b_{\nu_{i}}=b_{\nu_{k}} ;(\mathrm{b})$ if $\nu>\nu_{i}$, then either $a_{\nu}=\sum a_{\nu_{i}}$ and $b_{\nu}=\sum b_{\nu_{i}}$, or there exists a sequence $\left(\nu_{j}^{\prime}\right)$, such that $a_{\nu}-\sum a_{\nu_{i}}$ $=\sum a_{\nu_{j}^{\prime}}$ and $b_{\nu}-\sum b_{v_{i}}=\sum b_{v_{i}^{\prime}}$. (c) For corresponding subsequences $\left(a_{v_{i}}\right)$, $\left(b_{\nu_{i}}\right)$ of $\left(a_{\nu}\right)$ and $\left(b_{\nu}\right)$ there exists a sequence $\left(\nu_{j}^{\prime}\right)$ of subscripts such that $\left(a_{v_{j}^{\prime}}^{\prime}\right),\left(b_{\nu_{j}}\right)$ are disjoint and $\sum a_{\nu_{i}}=\sum a_{\nu_{i}^{\prime}}, \sum b_{\nu_{i}}=\sum b_{\nu_{j}^{\prime}}$.

12.5. Definition. Any (finite) union of base elements of any a of $M$ is called a finite $\sigma$-element of the basis $\left(a_{v}\right)$. The $\sigma$-element $\sum a_{\nu_{i}}$ will be denoted by $s_{\left[\nu_{i}\right]}(a)$.

From the previous results it follows that the $\sigma$-elements of any basis $\left(a_{v}\right)$ of an element $a$ have the properties listed below:

12.6. All finite products, all countable sums, and the relative complement of a finite $\sigma$-element with respect to any $\sigma$-element are all either $\sigma$-elements or equal to $\theta$. All $\sigma$-elements can be expressed as the sum of a sequence of disjoint base elements.

12.7. Any element $b<a$ is the product of a nested sequence of $\sigma$-elements $s_{k}(a)$ such that $\lim \left|s_{k}(a)\right|=|b|$.

For from the definition of a basis, for any sequence of positive numbers $\epsilon_{n} \rightarrow 0$, there exists a sequence of $\sigma$-elements $s_{n}(a)>b$, and such that $\left|s_{n}-b\right|<\epsilon_{n}$. But from 12.6, the elements $\prod_{i=1}^{k} s_{n}$ are $\sigma$-elements and satisfy the required conditions. 
12.8. If $\left(a_{\nu}\right)$, and $\left(a_{\nu}^{\prime}\right)$ are bases of $a$ and $a^{\prime}$, respectively, and if the corresponding $\sigma$-elements $s_{\alpha}(a) \leqq \sum a_{\nu_{i}(\alpha)}$ and $s_{\alpha}\left(a^{\prime}\right) \equiv \sum a_{\nu_{i}(\alpha)}$ are denoted by $s_{\alpha}$ and $s_{\alpha}^{\prime}$, then, for any sets of corresponding $\sigma$-elements $s_{i}$ and $s_{i}^{\prime}$ either both members of any one of the pairs $\prod_{i}^{k} s_{i}$ and $\prod_{i}^{k} s_{i}^{\prime}$ (where $k$ is any finite number), $\sum s_{i}$ and $\sum s_{i}^{\prime}$, and the pair of symmetric differences $s_{1}-s_{2}$ and $s_{1}^{\prime}-s_{2}^{\prime}$, are equal to $\theta$, or in each case there exists a pair of corresponding $\sigma$-elements $s_{\alpha_{1}}$ and $s_{\alpha_{1}}{ }^{\prime}$, or $s_{\alpha_{2}}$ and $s_{\alpha_{2}}^{\prime}$, or $s_{\alpha_{3}}$ and $s_{\alpha_{3}}^{\prime}$, such that $\prod_{i}^{k} s_{i}=s_{\alpha_{1}}$ and $\prod_{i}^{k} s_{i}^{\prime}=s_{\alpha_{1}}^{\prime}$, or $\sum s_{i}=s_{\alpha_{2}}$ and $\sum s_{i}^{\prime}=s_{\alpha_{2}}^{\prime}$, or $s_{1}-s_{2}=s_{\alpha_{3}}$ and $s_{1}^{\prime}-s_{2}^{\prime}=s_{\alpha_{3}}$.

\section{Infinite products. Regular bases. We prove first}

13.1. LEMMA. If $\left(a_{v_{i}}\right)$ is any infinite sequence of base elements with distinct subscripts, then $\prod a_{v_{i}}=\theta$.

Proof. We may assume that the sequence can be arranged so that $\nu_{i}\left\langle\nu_{i-1}\right.$, and so that $a_{\nu_{i}}<a_{\nu_{i-1}}$, for otherwise from (a) of 12.3 the conclusion that $\prod a_{\nu_{i}}=\theta$ already holds. If under this restriction $\prod a_{\nu_{i}}=a^{\prime} \neq \theta$, there exists a $\nu_{0}$ such that $\left|a_{\nu_{0}} a^{\prime}\right| \neq 0$. Then since for all $i, a_{\nu_{0}} a_{\nu_{i}} \neq \theta$, it follows for all but the finite set of the $a_{\nu_{i}}$ for which $n\left(\nu_{i}\right)<n\left(\nu_{0}\right)$, that $a_{\nu_{0}}<a_{\nu_{i}}$, and so further that $a_{\nu_{0}}>a^{\prime}$. By iii of $(\zeta)$, since $a^{\prime} \neq \theta$, there exists an $a^{\prime \prime}<a^{\prime}$ such that $\theta<a^{\prime \prime}<a^{\prime}$. But every $\sigma$-element $s$ covering $a^{\prime \prime}$ must also contain $a^{\prime}$, and hence $\left|s-a^{\prime \prime}\right|$ $>\left|s-a^{\prime}\right|>0$, which contradicts iv of $(\zeta)$. Therefore $a^{\prime}=\theta$.

13.2. Leмma. If $\left(a_{v}\right)$ is a basis of a satisfying 12.2, for any finite positive $\xi<|a|$ there exists a $\sigma$-element s such that $|s|=\xi$. More specifically, to any such $\xi$ we can make correspond a unique $\sigma$-element $s_{\xi}$ such that $\left|s_{\xi}\right|=\xi$, and if $0<\xi_{1}<\xi_{2} \leqq|a|, s_{\xi_{1}}<s_{\xi_{2}}$. Hence further each $\sigma$-element corresponds to at most one number $\xi$.

Proof. Since, for any $n, \sum a_{\nu}=a$, where $\nu \in \Gamma_{n}$, if we order the $a_{\nu}$ of $\Gamma_{n}$ according to the first differences into a sequence $a_{n, k}$ and if we let $s_{n, k}=\sum_{1}^{n} a_{n, i}$ then for each $\xi>0$ there corresponds a $k=k(\xi, n)$ such that

$$
\left|s_{n, k}\right| \leqq \xi \leqq\left|s_{n, k+1}\right| \text {, }
$$

and further

$$
s_{n, k_{n}}<s_{n+1, k_{n+1}}<s_{n+1, k_{n}+1}<s_{n, k_{n}+1} .
$$

From (1) and the fact that the elements $a_{n, \dot{k}}$ are nested, we have

$$
\xi-\left|\lim s_{n, k}\right|=\lim \left|a_{n, k}\right|=0 .
$$

Therefore $\left|\sum s_{n, k}\right|=\xi$ and since if $\xi_{1}<\xi_{2}$ then for each $n k_{n, \xi_{1}}<k_{n, \xi_{2}}$, we also have $\sum s_{n, k\left(\xi_{1}\right)}<\sum s_{n, k\left(\xi_{2}\right)}$, and we can define $s_{\xi}=\sum s_{n, k_{n, \xi}}$.

13.21. Lemma. Every element a such that $|a|=\infty$, is the sum of $a$ set of disjoint elements $a_{n}$ such that $\left|a_{n}\right|=1$ for each $n$.

Proof. Every such element $a$ is the sum of a set of elements $a_{n}^{\prime}$ such that 
$\left|a_{n}^{\prime}\right|<\infty$, and which we may suppose disjoint and different from $\theta$. Let $k_{i}$ be a monotonic set of integers such that $\left|a_{k_{i}}\right| \leqq i<\left|a_{k_{i+1}}\right|$, and let us define a nested sequence of elements $a_{i}^{*}$ as follows: If the lower inequality holds, let $a_{i}^{*}=a_{k}^{\prime}$; if $i>\left|a_{k_{i}}^{\prime}\right|$, let $a_{i}^{*}=a_{k+a^{\prime \prime}{ }_{i}}$, where $a_{i}^{\prime \prime} \leqq a_{k_{i+1}}^{\prime}-a^{\prime} k_{i}$, and $\left|a_{i}^{\prime \prime}\right|=i$ $-\left|a_{k_{i}}^{\prime}\right|$. In either case, $\left|a_{i}^{*}\right|=i$, and $a_{k_{i}} \leqq a_{i}^{*}<a_{k_{i+1}}$, whence $\lim a_{i}^{*}=a$. We can therefore define the required elements $a_{n}$ as $a_{1}{ }^{*}, a_{n}{ }^{*}-a_{n-1}^{*}$.

13.3. Definition. Let $\nu$ be any number of the form $k+m / 2^{n}$, where $k$ is $a$ positive integer, $0 \leqq m<2^{n}$, and $m / 2^{n}$ is expanded dyadically into a sequence of $n(\nu)$ terms. Then a basis $\left(a_{\nu}\right)$ of an element $a \neq \theta$ is called regular when $|a|<\infty$ if $0 \leqq \nu<1$ and $\left|a_{\nu}\right|=|a| / 2^{n(\nu)}$, and when $|a|=\infty$ if $0 \leqq \nu<\infty$ and $\left|a_{\nu}\right|=1 / 2^{n(\nu)}$.

\subsection{Theorem. Every $a \neq \theta$ has a regular basis.}

Proof. Suppose $|a|<\infty$, and let $\left(a_{\nu}^{\prime}\right)$ be any basis satisfying 12.2. Then there exist $\sigma$-elements $s_{n, m}$ for $n=1,2, \cdots$, and $m=1,2, \cdots, 2^{n}$, such that $\left|s_{n, m}\right|=m|a| / 2^{n}$, and such that $m / 2^{n}>m^{\prime} / 2^{n^{\prime}}$ implies that $s_{n, m}>s_{n^{\prime}, m^{\prime}}$. Then if $\nu$ is the dyadic expansion of $m / 2^{n}, m<2^{n}$, then we define $a_{\nu}$ as $s_{n, m+1}-s_{n, m}$, and we make the convention that $a_{0}=0$. If $s_{n, m}^{\prime}=\sum a_{\nu_{i}}^{\prime}$, where $\nu_{i}$ are the dyadic expansions of the numbers $m^{\prime} / 2^{n}, m^{\prime} \leqq m<2^{n}$, then $a_{\nu}^{\prime}=s_{n, m+1}^{\prime}-s^{\prime}{ }_{n, m}$ $=\sum a_{v_{j}}$, where $\left|s_{n, m}^{\prime}\right| \leqq \nu_{j} \leqq\left|s_{n, m+1}\right|$. Therefore from 12.1, $\left(a_{v}\right)$ is a basis, which can easily be seen to be regular. If $|a|=\infty$, let $\left(a_{k}\right)$ be a sequence satisfying 13.21, and combine their bases for a basis for $a$.

13.5. If $|a|=\left|a^{\prime}\right| \neq 0$, and if $\left(a_{\nu}\right),\left(a_{\nu}^{\prime}\right)$ are regular bases of $a$ and $a^{\prime}$, respectively, and if as previously we denote the corresponding $\sigma$-elements $s_{\alpha}(a)=\sum a_{\nu_{i}(\alpha)}$ and $s_{\alpha}^{\prime}=\sum a_{\nu_{i}(\alpha)}^{\prime}$ by $s_{\alpha}$ and $s_{\alpha}^{\prime}$ respectively, then for any set of corresponding pairs it follows from 12.8 and from the fact that $\left|a_{\nu}\right|=\left|a_{\nu}^{\prime}\right|$ that $\left|s_{\alpha}\right|=\left|s_{\alpha}^{\prime}\right| ;\left|s_{1}-s_{2}\right|=\left|s_{1}^{\prime}=s_{2}^{\prime}\right|$, where the differences are symmetric; $\left|\sum s_{i}\right|$ $=\left|\sum s_{i}^{\prime}\right|$; and $\left|\prod_{i}^{k} s_{i}\right|=\left|\prod_{i}^{k} s_{i}^{\prime}\right|$.

14. The isomorphism $\phi$. We are now in a position to establish the theorem referred to in $\$ 12$, namely, that

14.1. Theorem. If $|a|=\left|a^{\prime}\right|$, then there exists a $\sigma$-isomorphism $\phi$ of $I(a)$ (see 1.1) onto $I\left(a^{\prime}\right)$ such that for any $b \leqq a,|\phi(b)|=|b|$.

Proof. The theorem is trivial if $a=a^{\prime}=\theta$. Otherwise, let $\left(a_{\nu}\right),\left(a_{\nu}^{\prime}\right)$, be regular bases of $a$ and $a^{\prime}$ respectively. Then by 12.8 for any $b \leqq a$ there exists a nested sequence of $\sigma$-elements $s_{k}>b$ such that lim $\left|s_{k}-b\right|=0$. We define for any $b<a$.

$$
\phi(b) \equiv \phi\left(\prod s_{k}\right) \equiv \prod \phi\left(s^{\prime}{ }_{k}\right)
$$

Obviously, $\phi$ assigns at least one image to every element of $I(a)$ and at least one counterimage to every element of $I\left(a^{\prime}\right)$. Further from 13.5 for each $k$, $\left|s_{k}\right|=\left|s_{k}^{\prime}\right|$, whence for any image $\phi(b)$

$$
|\phi(b)|=\lim \left|s_{k}^{\prime}\right|=\lim \left|s_{k}\right|=|b|
$$


and the functional $|a|$ is invariant under $\phi$. Also, if $b_{1} \equiv \prod s_{k, 1}$ and $b_{2} \equiv \prod s_{k, 2}$ are any two elements of $I(a)$, then for each $k,\left|s_{k, 1}-s_{k, 2}\right|=\left|s_{k, 1}^{\prime}-s^{\prime}{ }_{k, 2}\right|$ where the differences are symmetric. Passing to the limit with respect to $k$,

$$
b_{1}=b_{2} \text { if and only if } \phi\left(b_{1}\right)=\phi\left(b_{2}\right),
$$

that is, the isomorphism $\phi(b)$ is univocal.

Now suppose $b_{1} \equiv \prod s_{k, 1} \leqq b_{2} \equiv \prod s_{k, 2}$. Then from 12.8 , the elements $s_{k, 3}=s_{k, 1} s_{k, 2}$ form a nested sequence of $\sigma$-elements such that $b_{1} \leqq s_{k, 3} \leqq s_{k, 2}$. Again from 13.5 for each $k, s_{k, 3}^{\prime}=s_{k, 1}^{\prime} s_{k, 2}^{\prime}$ so

$$
\phi\left(b_{1}\right)=\prod s_{k, 3}^{\prime} \leqq \prod s_{k, 2}^{\prime}=\phi\left(b_{2}\right) .
$$

Applying this same argument with reference to $\phi^{-1}$, we see that

$$
b_{1} \leqq b_{2} \text { if and onl } y \text { if } \phi\left(b_{1}\right) \leqq \phi\left(b_{2}\right) .
$$

Let $\left(a_{r}\right)$ be a sequence of elements of $I(a)$, and for each $r$ let $\left(s_{k, r}\right)$ be a nested sequence of $\sigma$-elements such that $a_{r}=\prod_{k} s_{k, r}$ and such that $\left|s_{k, r}-a_{r}\right|$ $<1 / 2^{k+r}$. Then for each $k, \sum_{r} s_{k, r}$ is a $\sigma$-element and

and so

$$
\begin{aligned}
& \sum a_{r} \leqq \sum_{r} s_{k, r} \leqq \sum_{r} s_{k-1, r}, \\
& \left|\sum_{r} s_{k, r}-\sum a_{r}\right|=\left|\sum_{r}\left[s_{k, r}-\left(\sum_{r} a_{r}\right) s_{k, r}\right]\right| \\
& \leqq \sum_{r}\left|s_{k, r}-a_{r}\right| \leqq 1 / 2^{k} \text {, }
\end{aligned}
$$

$$
a_{r}=\prod_{k}\left(\sum_{r} s_{k, r}\right) .
$$

From (5), for each $r, \phi\left(a_{r}\right) \leqq \phi\left(\sum a_{r}\right)$, and so further

$$
\sum \phi\left(a_{r}\right) \leqq \phi\left(\sum a_{r}\right) \text {. }
$$

But for each $k$,

$$
\begin{aligned}
\left|\sum_{r} s_{k, r}^{\prime}-\sum \phi\left(a_{r}\right)\right| & \leqq\left|s_{k, r}^{\prime}-\phi\left(a_{r}\right)\right|=\sum\left[\left|s_{k, r}^{\prime}\right|-\left|\phi\left(a_{r}\right)\right|\right] \\
& =\sum\left(\left|s_{k, r}\right|-\left|a_{r}\right|\right) \leqq 1 / 2^{k}
\end{aligned}
$$

and passing to the limit with respect to $k$ gives

$$
\sum \phi\left(a_{r}\right)=\phi\left(\sum a_{r}\right) \text {. }
$$

From 12.6 for each finite $r$ the elements $\prod_{0}^{r} s_{k, i}$ form a nested sequence of $\sigma$-elements such that $\prod_{0}^{\infty} a_{i} \leqq \prod_{0}^{r} a_{i} \leqq \prod_{0}^{r} s_{k, i}$. If for some $i\left|a_{i}\right|<\infty$, and if $\left(r_{i}\right)$ is an increasing sequence of indices such that $\left|\prod_{0}^{r_{i}} a_{r}\right|-\left|\prod_{0}^{\infty} a_{r}\right|<1 / 2 i$, 


$$
\left|\prod_{0}^{r_{i}} s_{k, r}-\prod_{0}^{\infty} a_{k, r}\right| \leqq\left|\prod_{0}^{r_{i}} s_{k, r}-\prod_{0}^{r_{i}} a_{r}\right|+\left|\prod_{0}^{r_{i}} a_{r}-\prod_{0}^{\infty} a_{r}\right| \cdot
$$

But since $s_{k, r} \leqq \prod_{0}^{r_{i}} a_{r}+\sum_{0}^{r_{i}}\left(s_{k, r}-a_{r}\right)$, (12) yields

$$
\left|\prod_{0}^{r_{i}} s_{k, r}-\prod_{0}^{\infty} a_{r}\right|<1 / 2^{k+i}
$$

whence

$$
\prod_{0}^{\infty} a_{r}=\prod_{k=0}^{\infty}\left(\prod_{r=0}^{r k} s_{k, r}\right)
$$

From (5), for each $r, \phi\left(\prod_{r} a_{r}\right) \leqq \phi\left(a_{r}\right)$, and so

$$
\phi\left(\prod a_{r}\right) \leqq \prod \phi\left(a_{r}\right) .
$$

On the other hand,

$$
\sum \phi\left(a_{r}\right)=\prod_{r=0}^{\infty} \prod_{k=0}^{\infty} s_{k, r}^{\prime}=\prod_{k=0}^{\infty} \prod_{r=0}^{\infty} s_{k, r}^{\prime} \leqq \prod_{k=0}^{\infty} \prod_{r=0}^{r k} s_{k, r}^{\prime}=\phi\left(\prod a_{r}\right),
$$

which with (15) gives

$$
\prod \phi\left(a_{r}\right)=\phi\left(\prod a_{r}\right),
$$

if for some $i,\left|a_{i}\right|<\infty$. If for all $r,\left|a_{r}\right|=\infty$, let $\left(a_{1, j}\right)$ be a sequence of elements such that $\sum a_{1, j}=a_{1}$ and $\left|a_{1, j}\right|<\infty$ for all $j$ 's. Then from (11) and (17),

$$
\phi\left(\prod a_{r}\right)=\phi\left[\sum_{j}\left(\prod_{r} a_{1, j} a_{r}\right)\right]=\sum_{j}\left[\prod_{r} \phi\left(a_{1, j}\right) \phi\left(a_{r}\right)\right]=\prod \phi\left(a_{r}\right),
$$

and this holds for any sequence $\left(a_{r}\right)$.

It is now verified by statements (2), (3), (11), and (18) that $\phi$ satisfies the conditions required in Theorem 14.1, the proof of which is now completed. We have as an immediate corollary to 14.1 that

14.11. The class $\Phi$ of all $\sigma$-isomorphisms $\phi$ between pairs of principal ideals of $M$ is non-void and satisfies $(\beta)$ of $\$ 1$.

\section{Application to Part II. Examples.}

15.1. If now $m$ is a field of sets upon which a measure function $|a|$ is defined, and if $n$ is the ideal of sets for which $|a|=0$, then we define a functional $|a|$ on the residue class ring $M=m / n$ as the common value of $|a|$ for all the sets $a \in a$. If $|a|$ is such that $(\zeta)$ holds, then by Theorem 15.1 we have a measuring system $(m, n, \Phi)(\$ 6)$. If as before we denote the measure in the sense of Part II of any $a \in M$ by $\mathfrak{a}$, then $\mathfrak{a}=\mathfrak{b}$ if and only if $|a|=|b|, \mathfrak{a}=0$ if and only if $a=\theta$, that is, if and only if $|a|=0$; and $a=\sum \mathfrak{a}_{n}$ if and only if $|a|=\sum\left|a_{n}\right|$. Further from 13.2, $\mathfrak{a}$ is bounded in the sense of Part II if, and from 15.21 only if, $|a|<\infty$. From the fact that $a<b$ if and only if $|a|<|b|$, 
it is clear that the system $(m, n, \Phi)$ satisfies $(\delta)(\S 9)$ and $(\epsilon)(\S 10)$. Therefore the correspondence $f(a) \equiv f(a)=|a|$ is an isomorphism of the type described in Theorem 10.1 of the set $\mathfrak{M}$ of measure values onto a closed interval. It follows from 10.1 that the ratios $|a| /|b|$ are uniquely determined by the system $(m, n, \Phi)$ as the numbers $\mu(a, b)$ of the lemma to 10.1 , and so that $|\boldsymbol{a}|$ is determined up to a multiplicative constant.

15.2. EXAmpLEs. Let $s$ be any locally compact metric spaces and let $|a|$ be a positive functional on the open sets with compact closures such that $|a|=0$ only on the empty set, and such that with any set of concentric spheres $a_{\delta}$ of radius $\epsilon, \lim \left|a_{\epsilon}\right|=0$. Then the measure generated in the usual fashion from $|a|$ satisfies conditions $(\zeta)$ and so on can be obtained as in Part II. The Haar measure on a locally compact group is also covered by Part II, since the decomposition theorem of Banach and Tarski can be extended $\left.{ }^{3}\right)$ to this case; if the fundamental space is not a group, but metric and locally compact $\left({ }^{4}\right)$, we need make only a trivial examination of the case in which points have positive measure.

15.3. The theorem of Banach and Tarski mentioned in the Introduction can be generalized under conditions of 15.1 in the following sense: Let $\left(s_{\nu}\right)$ be a regular basis of $s(13.3)$. An isomorphism $\phi$ of $\Phi$ is called a congruence if $\phi$ is defined between two base elements, say $s_{\nu}$ and $s_{\nu^{\prime}}$, where obviously $n(\nu)=n\left(\nu^{\prime}\right)$, and satisfies the following condition:

Let $\nu, \nu^{\prime}$, be the dyadic expansions of $m / 2^{n}, m^{\prime} / 2^{n}$, respectively. The elements $s_{\gamma}<s_{\nu}\left[s_{\gamma^{\prime}}<s_{\nu}\right]$ are those such that $m / 2^{n} \leqq \gamma<m+1 / 2^{n},\left[m^{\prime} / 2^{n} \leqq \gamma^{\prime}\right.$ $\left.<m^{\prime}+1 / 2^{n}\right]$. We define $\phi\left(s_{\gamma}\right)$ for all such $\gamma$ as $s_{\gamma^{\prime}}$, where $\gamma^{\prime}=m^{\prime} / 2^{n}+\left(\gamma-m / 2^{n}\right)$. Obviously, $\phi$ is completely determined for any $s^{\prime}<s$ by the above relation. Then under conditions $(\zeta)$ and with this definition for congruence, a necessary and sufficient condition that two elements $a$ and $a^{\prime}$ be of equal measure is that there exist sequences $\left(a_{n}\right),\left(a_{n}^{\prime}\right)$ of disjoint elements such that $a=\sum a_{n}$, $a^{\prime}=\sum a_{n}^{\prime}$ and $a_{n}$ and $a_{n}^{\prime}$ are congruent for each $n$.

The sufficiency is obvious. The necessity will be proved for the case for which $|a|=\left|a^{\prime}\right|<\infty$ and both $a$ and $a^{\prime}$ are different from $s$, from which the general case follows at once. The required proof then reduces to that of the following statements: a. If $|a|=\left|a^{\prime}\right|<\infty$, then each element $a$ or $a^{\prime}$ is the limit of a nested sequence of $\sigma$-elements of finite measure, of which corresponding elements have equal measure. b. Each of two $\sigma$-elements of equal measure is the sum of a family of disjoint base elements, corresponding elements having equal measure and so being congruent. For if a and $b$ hold, and if we let the class of all sums of congruences, which class can be seen to satisfy $(\beta)$, be $\Phi$, then we can apply Theorem 4.11 (which states that the respective limits of

(3) See Footnote 1.

(4) As in the "Note on Haar's measure," by S. Banach, in Saks' Theory of the Integral, Appendix II. 
monotonic sequences of bounded equivalent elements are equivalent) and so get the desired theorem.

To prove a, we consider nested sequences of $\sigma$-elements $s_{k}>a$, and $s_{k}^{\prime}>a^{\prime}$, such that for some $\epsilon>0,|s|>\epsilon \geqq\left|s_{k}-a\right| \geqq 2\left|s_{k+1}-a\right|$, and $\left|s_{k}^{\prime}-a^{\prime}\right|<\left|s_{k}-a\right|$. It follows that $\left(\left|s_{k}\right|-\left|s_{k}^{\prime}\right|\right)<\left(\left|s_{k-1}\right|-\left|s_{k-1}^{\prime}\right|\right)$. But for each $p$, the elements $\left(s_{\nu}\right)$ for which $n(\nu)=p$ can be ordered into a sequence $\left(s_{n, p}\right)$. Then for each $k$ and $p$, there is an integer $i=i_{k, p}$ for which $\left|\sum_{j=0}^{i-1} s_{j, p}+s_{k}^{\prime}\right| \leqq\left|s_{k}\right|-\left|s_{k}^{\prime}\right|$ $<\left|\sum_{j=0}^{i} s_{j, p}+s_{k}^{\prime}\right|$, and the proof proceeds as in 13.2. Relation $\mathrm{b}$ is obvious in the case of two finite sums of base elements, for each can be expressed as the sum of elements $s_{\gamma}$ such that $n(\nu)=n^{*}$, where $n^{*}$ is the maximum value of $n(\nu)$ occurring in the given summands. Otherwise we write both summands as infinite sums. But two infinite sums can, as a result of 13.21 and the regularity of the basis $\left(s_{\nu}\right)$, be expressed as sums of infinite disjoint sets of finite sums, of which corresponding finite sums have equal measure. Hence our assertion is proved.

In the case of euclidean $r$-space $\left(\xi_{1}, \cdots, \xi_{r}\right)$ we may consider our class $\Phi$ as sums of rigid motions and the regular basis as the family of cubes $\left[m / 2^{n} \leqq \xi_{i}<(m+1) / 2^{n}\right], i=1, \cdots, r$. Our result then reduces to the theorem of Banach and Tarski from the fact that every open set is the union of a set of disjoint half-open cubes, and that every measurable set is $G_{\delta}$, modulo the null sets.

It can be deduced from the results of Part III that under the conditions of 15.1 that $M=m / n$ and the totality $\Phi$ of measure-preserving transformations on $M$ are respectively isomorphic to the field $M^{\prime}$ of measurable sets modulo the null sets in euclidean space, and to the totality $\Phi^{\prime}$ of measurepreserving transformations on $M^{\prime}$.

Bryn Mawr College, BRYN MAWR, Pa. 